\title{
WAVE ENHANCEMENT THROUGH OPTIMIZATION OF BOUNDARY CONDITIONS*
}

\author{
HABIB AMMARI ${ }^{\dagger}$, OSCAR BRUNO ${ }^{\ddagger}$, KTHIM IMERI $^{\dagger}$, AND NILIMA NIGAM ${ }^{\S}$
}

\begin{abstract}
In this paper, we present a new and efficient approach for optimizing the transmission signal between two points in a cavity at a given frequency, by changing boundary conditions. The proposed approach makes use of recent results on the monotonicity of the eigenvalues of the mixed boundary value problem and on the sensitivity of Green's function to small changes in the boundary conditions. The switching of the boundary condition from Dirichlet to Neumann can be performed through the use of the recently modeled concept of metasurfaces which are comprised of coupled pairs of Helmholtz resonators. A variety of numerical experiments are presented to show the applicability and the accuracy of the proposed new methodology.
\end{abstract}

Key words. Zaremba eigenvalue problem, boundary integral operators, mixed boundary conditions, metasurfaces

AMS subject classifications. 35R30, 35C20

DOI. $10.1137 / 19 \mathrm{M} 1274651$

1. Introduction. This paper develops a new and efficient approach for maximizing the transmission signal between two points at a chosen frequency through changes to specific eigenmodes of the cavity. These changes are achieved by changing the boundary conditions. The eigenmodes and the associated eigenfrequencies of a cavity are sensitively dependant on the geometric properties of the domains, as well as the location of Dirichlet and Neumann boundary conditions. Many recent works have been devoted to the understanding of the effect of changing the boundary condition on the eigenmodes and the eigenfrequencies $[1,2,3,6,12,15,16,18]$.

Through the use of a tunable reflecting metasurface, the boundary condition can be switched from Dirichlet to Neumann at some specific resonant frequencies [4]. In [4], the physical mechanism underlying the concept of tunable metasurfaces is modeled both mathematically and numerically. It is shown that an array of coupled pairs of Helmholtz resonators behaves as an equivalent surface with Neumann boundary condition at some specific subwavelength resonant frequencies, where the size of one pair of Helmholtz resonators is much smaller than the wavelengths at the resonant frequencies. Green's function of a cavity with mixed (Dirichlet and Neumann) boundary conditions (called also the Zaremba function) is also characterized. In [5], a one-shot optimization algorithm is proposed and used to obtain a good initial guess for the positions around which the boundary conditions should be switched from Dirichlet to Neumann.

*Submitted to the journal's Computational Methods in Science and Engineering section July 15, 2019; accepted for publication (in revised form) December 9, 2019; published electronically February 4, 2020.

https://doi.org/10.1137/19M1274651

$\dagger$ Department of Mathematics, ETH Zürich, Rämistrasse 101, CH-8092 Zürich, Switzerland (habib.ammari@sam.math.ethz.ch, kthim.imeri@sam.math.ethz.ch).

${ }^{\ddagger}$ Computing \& Mathematical Sciences, California Institute of Technology, 1200 E California Blvd., Pasadena, CA 91125 (obruno@caltech.edu).

$\S$ Department of Mathematics, Simon Fraser University, 8888 University Dr., Burnaby, BC V5A 1S6, Canada (nigam@math.sfu.ca).

B207 
The existing approach for maximizing the transmission between two points inside the cavity is the following [13]. One divides the boundary into small parts and starts with a uniform Dirichlet condition. Then, one iteratively switches on each part of the boundary the boundary condition from Dirichlet to Neumann and computes each time the transmission signal between the chosen points inside the cavity. If the transmission is higher, the Neumann boundary condition is kept; otherwise, it is switched back to Dirichlet. Finally, this algorithm is iterated $n$ times and is therefore very timeconsuming.

In this paper, we present a new and very efficient methodology for maximizing the Zaremba function between two points at a chosen frequency through specific eigemodes of the cavity. The paper is organized as follows. In section 2, we first recall some useful results on the eigenvalues of the mixed boundary value problem (called the Zaremba eigenvalue problem). Of particular interest is the monotonicity property with respect to the size of the Neumann part proven in [16]. Then we reformulate the eigenvalue problem using boundary integral operators. Based on this nonlinear formulation and the use of the generalized argument principle for the characterization of the characteristic values of finitely meromorphic operator-valued functions of Fredholm type, we derive an accurate asymptotic formula of the changes of eigenfrequencies of a cavity with mixed boundary conditions in terms of the size of the part of the cavity boundary where the boundary condition is switched from Dirichlet to Neumann. Finally, we recall the asymptotic expansion of the Zaremba function in terms of the size of the Neumann part. The problem of changing a portion of a Dirichlet boundary to Neumann is more delicate than the converse. If a portion of the boundary is changed from having Neumann conditions to Dirichlet, the reverse consideration of that in this paper, then an asymptotic expansion of the eigenvalues is easier to derive $[6,18]$. The perturbation theory for the introduction of Neumann boundaries requires a careful consideration of the asymptotic behavior of the Zaremba near the perturbation [5]. In section 3, we derive a spectral decomposition of the Zaremba function. In section 4 , we consider the problem where we have a source in a bounded domain operating at a given frequency, and we want to determine, by exploiting the monotonicity property of the eigenvalues of the mixed boundary value problem, which part of the boundary to choose to be reflecting such that an eigenvalue of the mixed boundary value problem gets close enough to the operating frequency. In order to significantly enhance the signal at a given receiving point, both the emitter and the receiver should not belong to the nodal set corresponding to the eigenmode associated with the eigenvalue of the mixed boundary value problem.

There are two distinct issues: where to place the Neumann boundary condition, and how long it should be, to achieve the twin objectives of maximizing gain between a fixed source-receiver pair and at a frequency close to a desired one.

Our main idea is to first nucleate the Neumann boundary conditions in order to maximize gain of the Zaremba function by making use of an asymptotic expansion of the Zaremba function in terms of the size of the Neumann part. Then the size of the Neumann part is changed in such a way that an eigenvalue of the mixed boundary value problem gets close to the operating frequency by using the monotonicity property of the eigenvalues of the mixed eigenvalue problem. The optimization needs the high-accuracy evaluation of certain boundary integral operators, and this is done using techniques from $[1,2]$. In view of the spectral decomposition of the Zaremba function in Theorem 3.1, the transmission between the given points at the chosen frequency gets very much enhanced.

We present in section 5 some numerical experiments to show the applicability and the accuracy of the proposed methodology. 


\section{Preliminaries.}

2.1. Laplace eigenvalue with mixed boundary conditions. Let $\Omega \subset \mathbb{R}^{2}$ be an open, bounded domain with a smooth boundary. We define $\bar{\Omega}$ as the topological closure of $\Omega$. We decompose the boundary $\partial \Omega:=\bar{\Omega} \backslash \Omega$ into two parts, $\partial \Omega=\overline{\Gamma_{\mathrm{D}} \bullet \Gamma_{\mathrm{N}}}$, where $\Gamma_{\mathrm{D}}$ and $\Gamma_{\mathrm{N}}$ are finite unions of open boundary sets and $\bullet$ denotes the disjoint union (see Figure 2.1). We define $\left(\Gamma_{\mathrm{D}}, \Gamma_{\mathrm{N}}\right)$ to be a partition of $\partial \Omega$. Let $x_{\mathrm{S}} \in \Omega$ and $k \in(0, \infty)$. The Zaremba function $\mathrm{Z}_{x_{\mathrm{S}}}^{k}\left(x_{s}, \cdot\right): \Omega \backslash\left\{x_{\mathrm{S}}\right\} \rightarrow \mathbb{R}$ is Green's function to the Zaremba problem, also known as the fundamental Helmholtz equation with mixed boundary conditions,

$$
\left\{\begin{array}{cl}
\left(\triangle+k^{2}\right) \mathrm{Z}_{x_{\mathrm{S}}}^{k}\left(x_{\mathrm{S}}, y\right)=\delta_{0}\left(x_{\mathrm{S}}-y\right) & \text { for } \quad y \in \Omega, \\
\mathrm{Z}_{x_{\mathrm{S}}}^{k}\left(x_{\mathrm{S}}, y\right)=0 & \text { for } \quad y \in \Gamma_{\mathrm{D}} \\
\partial_{\nu_{y}} \mathrm{Z}_{x_{\mathrm{S}}}^{k}\left(x_{\mathrm{S}}, y\right)=0 & \text { for } \quad y \in \Gamma_{\mathrm{N}} .
\end{array}\right.
$$

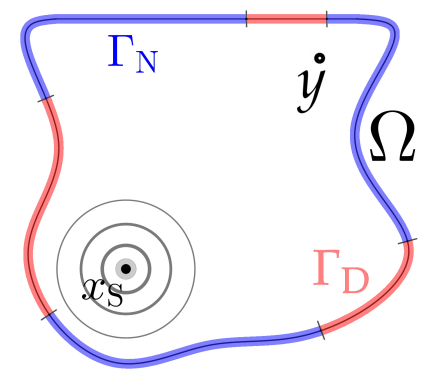

FIG. 2.1. $\Gamma_{N}$ is marked in blue and $\Gamma_{D}$ in red.

Here $\nu_{y}$ denotes the outer normal at $y \in \partial \Omega$ and $\partial_{\nu_{y}}$ the normal derivative at $y \in \partial \Omega$. It is clear that we can write

$$
Z^{k}\left(x_{s}, \cdot\right)=\Gamma^{k}\left(x_{s}, \cdot\right)+\mathrm{R}^{k}\left(x_{s}, \cdot\right),
$$

where $\Gamma^{k}(x, y):=\frac{i}{4} H_{0}^{1}(k|x-y|)$ is the fundamental solution of the Helmholtz problem with wavenumber $k$ and $\mathrm{R}^{k}\left(x_{s}, \cdot\right)$ is a smooth function satisfying the boundary value problem

$$
\left\{\begin{aligned}
\left(\triangle+k^{2}\right) \mathrm{R}^{k}\left(x_{s}, y\right) & =0 & & \text { in } \Omega, \\
\mathrm{R}^{k}\left(x_{s}, y\right) & =-\Gamma^{k}\left(x_{s}, y\right) & & \text { on } \Gamma_{D} \\
\partial_{\nu_{y}} \mathrm{R}^{k}\left(x_{s}, y\right) & =-\partial_{\nu_{y}} \Gamma^{k}\left(x_{s}, y\right) & & \text { on } \Gamma_{N}
\end{aligned}\right.
$$

In section 3, we will see that $\mathrm{Z}_{x_{\mathrm{S}}}^{k}$ exists for all but countably many values of $k$, which are related to the unique solvability of the problem for $\mathrm{R}^{k}\left(x_{\mathrm{S}}, \cdot\right)$. These exceptional values of $k$ are the eigenvalues to the associated Laplace eigenvalue problem with mixed boundary conditions

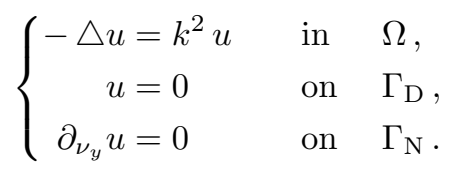


Equation (2.3) has a nontrivial solution $u \in \mathrm{H}^{1}(\Omega)$ for a countable set of real values of $k^{2}\left[17\right.$, Theorem 4.10], which we refer to as $\left\{\lambda_{j}^{\Gamma_{\mathrm{D}}}\right\}_{j=1}^{\infty}$, so that $\lambda_{1}^{\Gamma_{\mathrm{D}}} \leq \lambda_{2}^{\Gamma_{\mathrm{D}}} \leq \lambda_{3}^{\Gamma_{\mathrm{D}}} \leq \cdots$. We know that $\lambda_{1}^{\Gamma_{\mathrm{D}}} \geq 0$ and that $\lim _{j \rightarrow \infty} \lambda_{j}^{\Gamma_{\mathrm{D}}}=+\infty$ for all partitions $\left(\Gamma_{\mathrm{D}}, \Gamma_{\mathrm{N}}\right)$ of $\partial \Omega$.

We denote by $\left\{\lambda_{j}^{\partial \Omega}\right\}_{j \in \mathbb{N}}$ the pure Dirichlet eigenvalues for $\Omega$, corresponding to the case $\Gamma_{\mathrm{D}}=\partial \Omega$. We let $\left\{\lambda_{j}^{\varnothing}\right\}_{j \in \mathbb{N}}$ denote the Neumann eigenvalues associated to the case $\Gamma_{\mathrm{N}}=\partial \Omega$. Then we have

$$
\begin{array}{lll}
0<\lambda_{1}^{\partial \Omega}, & \lambda_{1}^{\partial \Omega}<\lambda_{2}^{\partial \Omega}, & \lambda_{2}^{\partial \Omega} \leq \lambda_{j}^{\partial \Omega} \forall j \geq 3, \\
0=\lambda_{1}^{\varnothing}, & \lambda_{1}^{\varnothing}<\lambda_{2}^{\varnothing}, & \lambda_{2}^{\varnothing} \leq \lambda_{j}^{\varnothing} \forall j \geq 3 .
\end{array}
$$

In [10], it is shown that $\lambda_{j+1}^{\varnothing}<\lambda_{j}^{\partial \Omega}$ for all $j \in \mathbb{N}$, for a very general class of domains $\Omega$.

Remark 2.1. Let $\Omega$ be the unit circle; we have that $\left\{\lambda_{j}^{\partial \Omega}\right\}_{j=1}^{\infty}$ is (up to sorting) equal to

$$
\left\{\alpha^{2} \in(0, \infty) \mid \exists n \in \mathbb{N}_{0}: \alpha \text { is positive root of } J_{n}(x)\right\},
$$

where $J_{n}$ is a Bessel function of the first kind and order $n$. The eigenvalues corresponding to the roots of $J_{0}$ appear as simple Dirichlet eigenvalues; all others have multiplicity two, and $\left\{\lambda_{j}^{\varnothing}\right\}_{j=2}^{\infty}$ is (up to sorting) equal to

$$
\left\{\alpha^{2} \in(0, \infty) \mid \exists n \in \mathbb{N}_{0}: \alpha \text { is positive root of } J_{n}^{\prime}(x)\right\} .
$$

Again, the eigenvalues corresponding to the roots of $J_{0}^{\prime}$ appear as simple Neumann eigenvalues; all others have multiplicity two. We refer to [11].

Recently, Lotoreichik and Rohleder [16, Proposition 2.3] showed the following monotonicity statement (see Figure 2.2).

Proposition 2.1. Let $\left(\Gamma_{D}, \Gamma_{N}\right),\left(\Gamma_{D}{ }^{\prime}, \Gamma_{N}{ }^{\prime}\right)$ be two partitions of $\partial \Omega$ such that $\Gamma_{D} \subset \Gamma_{D}^{\prime}$. If $\Gamma_{D}{ }^{\prime} \backslash \Gamma_{D}$ has a nonempty interior, then

$$
\lambda_{j}^{\Gamma_{D}}<\lambda_{j}^{\Gamma_{D^{\prime}}} \quad \forall j \in \mathbb{N} .
$$
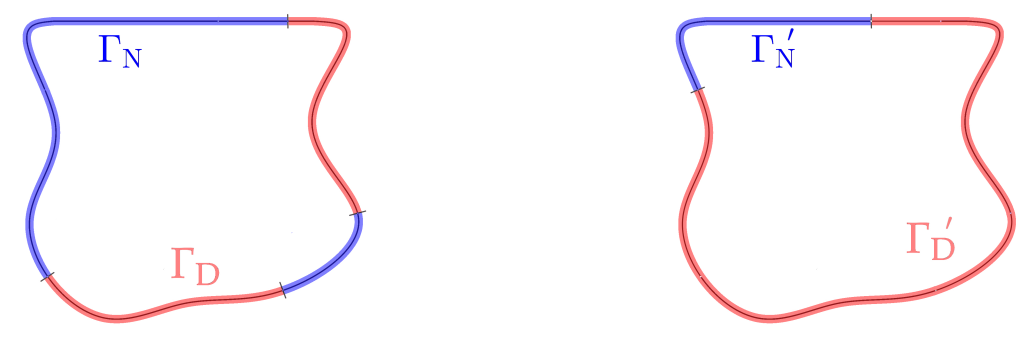

FIG. 2.2. An illustrative example of the two partitions mentionned in Proposition 2.1. On the left-hand side we have the parition $\left(\Gamma_{\Delta}, \Gamma_{N}\right)$ and on the right-hand side $\left(\Gamma_{\Delta}^{\prime}, \Gamma_{N}^{\prime}\right)$. They satisfy the condition $\Gamma_{\Delta} \subset \Gamma_{\Delta}^{\prime}$ and that $\Gamma_{\Delta}^{\prime} \backslash \Gamma_{\Delta}$ has a nonempty interior.

With Proposition 2.1, we can readily infer that if $\varnothing \neq \Gamma_{\mathrm{D}}$, and $\overline{\Gamma_{\mathrm{D}}} \neq \partial \Omega$, then

$$
\lambda_{j}^{\varnothing}<\lambda_{j}^{\Gamma_{\mathrm{D}}}<\lambda_{j}^{\partial \Omega} \quad \forall j \in \mathbb{N} .
$$

Copyright $@$ by SIAM. Unauthorized reproduction of this article is prohibited. 
2.2. Boundary integral formulation of the eigenvalue problem. The solution $u$ of the eigenvalue (2.3) can be represented by a single layer potential

$$
u(x)=\int_{\partial \Omega} \Gamma^{k}(x, y) \psi(y) \mathrm{d} \sigma_{y}
$$

with surface density $\psi \in L^{2}(\partial \Omega)$.

We define then the operators

$$
\begin{gathered}
\mathcal{S}_{\Gamma_{\mathrm{D}}}^{k}: H^{-1 / 2}\left(\Gamma_{\mathrm{D}}\right) \rightarrow H^{1 / 2}\left(\Gamma_{\mathrm{D}}\right), \quad \mathcal{S}_{\Gamma_{\mathrm{N}}}^{k}: H^{-1 / 2}\left(\Gamma_{\mathrm{N}}\right) \rightarrow H^{-1 / 2}\left(\Gamma_{\mathrm{D}}\right), \\
\left(\mathcal{K}_{\Gamma_{\mathrm{N}}}^{k}\right)^{*}: H^{-1 / 2}\left(\Gamma_{\mathrm{N}}\right) \rightarrow H^{-1 / 2}\left(\Gamma_{\mathrm{N}}\right), \text { and } \partial \mathcal{S}_{\Gamma_{\mathrm{D}}}^{k}: H^{-1 / 2}\left(\Gamma_{\mathrm{D}}\right) \rightarrow H^{-1 / 2}\left(\Gamma_{\mathrm{N}}\right) \text { by } \\
\mathcal{S}_{\Gamma_{\mathrm{D}}}^{k}[\psi](x):=\int_{\Gamma_{\mathrm{D}}} \Gamma^{k}(x, y) \psi(y) \mathrm{d} \sigma_{y}, \quad \mathcal{S}_{\Gamma_{\mathrm{N}}}^{k}[\psi](x):=\int_{\Gamma_{\mathrm{N}}} \Gamma^{k}(x, y) \psi(y) \mathrm{d} \sigma_{y}, \\
\partial \mathcal{S}_{\Gamma_{\mathrm{D}}}^{k}[\psi](x):=\int_{\Gamma_{\mathrm{D}}} \partial_{\nu_{x}} \Gamma^{k}(x, y) \psi(y) \mathrm{d} \sigma_{y}, \quad\left(\mathcal{K}_{\Gamma_{\mathrm{N}}}^{k}\right)^{*}[\psi](x):=\text { p. } \int_{\Gamma_{\mathrm{N}}} \partial_{\nu_{x}} \Gamma^{k}(x, y) \psi(y) \mathrm{d} \sigma_{y},
\end{gathered}
$$

where the "p.v." stands for the principle value integral. This actually is the standard (Lebesgue) integral for a smooth curved $\Gamma_{\mathrm{N}}$, since $\partial_{\nu} \Gamma^{k}$ is a bounded and sufficiently smooth integral operator kernel. From [19, Chapter 11] we have that $\mathcal{S}_{\Gamma_{\mathrm{D}}}^{k}$ is a Fredholm operator with index 0 . We also readily infer that $\left(\mathcal{K}_{\Gamma_{\mathrm{N}}}^{k}\right)^{*}, \partial \mathcal{S}_{\Gamma_{\mathrm{D}}}^{k}$, and $\mathcal{S}_{\Gamma_{\mathrm{N}}}^{k}$ are compact operators.

We then define $\mathcal{A}(k): H^{-1 / 2}\left(\Gamma_{\mathrm{D}}\right) \times H^{-1 / 2}\left(\Gamma_{\mathrm{N}}\right) \rightarrow H^{1 / 2}\left(\Gamma_{\mathrm{D}}\right) \times H^{-1 / 2}\left(\Gamma_{\mathrm{N}}\right)$ in terms of these integral operators through

$$
\mathcal{A}(k)\left[\begin{array}{l}
\left.\psi\right|_{\Gamma_{\mathrm{D}}} \\
\left.\psi\right|_{\Gamma_{\mathrm{N}}}
\end{array}\right]:=\left[\begin{array}{cc}
\mathcal{S}_{\Gamma_{\mathrm{D}}}^{k} & \mathcal{S}_{\Gamma_{\mathrm{N}}}^{k} \\
\partial \mathcal{S}_{\Gamma_{\mathrm{D}}}^{k} & -\frac{1}{2} \mathrm{I}_{L_{\omega}^{2}\left(\Gamma_{\mathrm{N}}\right)}+\left(\mathcal{K}_{\Gamma_{\mathrm{N}}}^{k}\right)^{*}
\end{array}\right]\left[\begin{array}{c}
\left.\psi\right|_{\Gamma_{\mathrm{D}}} \\
\left.\psi\right|_{\Gamma_{\mathrm{N}}}
\end{array}\right] .
$$

We readily see that $\mathcal{A}(k)$ is an analytic Fredholm operator of index 0 in $\mathbb{C} \backslash i \mathbb{R}^{-}$. To locate the Zaremba eigenvalues, we have the following statement:

The real positive characteristics values of the operator-valued function

$$
k \mapsto \mathcal{A}(k) \text { are the square roots of the Zaremba eigenvalues. }
$$

In [2, section 3] and [1], it is shown that every square root of a Zaremba eigenvalue is a real positive characteristic value of $k \mapsto \mathcal{A}(k)$ and every real positive characteristic value of $k \mapsto \mathcal{A}(k)$ is the square root of a Zaremba eigenvalue.

We see that $\mathcal{A}(k)$ is invertible for $k \in(0, \infty)$ not a square root of a Zaremba eigenvalue.

We remark that the nonreal characteristic values of $k \mapsto \mathcal{A}(k)$ cannot correspond to eigenvalues to the Laplace equation. This yields the undesirable, but avoidable, difficulty in choosing a neighborhood $V$ to apply Proposition 2.4 in our algorithm; see also section 4, comment on line 13 .

The statement (2.6) allows for a discretization and thus a numerical approximation of the value $k$. We will use this further on. For these facts, we refer to [2, sections 3 and 5].

Let us also consider the regularity of the solution $u$ and the density $\psi$ near a Dirichlet-Neumann junction. The following result (see Figure 2.3) can be found in [1, Theorems 4.2 and 4.3]. 


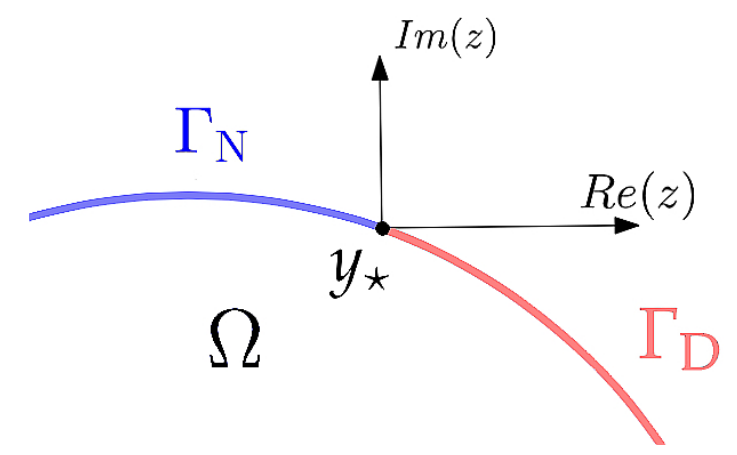

FIG. 2.3. An illustration of the setup used in Proposition 2.2. $z$ is defined as the complexification of a $\mathbb{R}^{2}$-vector with the origin at $y_{\star}$.

Proposition 2.2. Let $\Gamma_{D}, \Gamma_{N}$ be nonempty. Let $k>0$ and $\psi$ satisfy the statement (2.6). Let $y_{\star} \in \overline{\Gamma_{D}} \cap \overline{\Gamma_{N}}$. Then there exists a neighborhood $\mathcal{U} \subset \mathbb{R}^{2}$ around $y_{\star}$ such that for all $y \in \mathcal{U}$ and for all $n \in \mathbb{N}$,

$$
\begin{aligned}
u(y) & =\mathrm{P}_{y_{\star}}^{n}\left(z^{1 / 2}, \bar{z}^{1 / 2}\right)+o\left(z^{n}\right), \\
\left.\psi\right|_{\Gamma_{D}}(y) & =|z|^{-1 / 2} \mathrm{Q}_{\mathrm{D}, y_{\star}}^{n}\left(|z|^{1 / 2}\right)+o\left(|z|^{n-1}\right), \\
\left.\psi\right|_{\Gamma_{N}}(y) & =|z|^{-1 / 2} \mathrm{Q}_{\mathrm{N}, y_{\star}}^{n}\left(|z|^{1 / 2}\right)+o\left(|z|^{n-1}\right),
\end{aligned}
$$

where $z \in \mathbb{C}$ is the complexification of $y-y_{\star}$, that is, $z=\left(y_{1}-\left(y_{\star}\right)_{1}\right)+i\left(y_{2}-\left(y_{\star}\right)_{2}\right)$ with $i$ being the imaginary unit and $\bar{z}$ being its conjugate value. Here, $P_{y_{\star}}^{n}, \mathrm{Q}_{\mathrm{D}, y_{\star}}^{n}, \mathrm{Q}_{\mathrm{N}, y_{\star}}^{n}$ are polynomial functions of their respective arguments and of a degree such that none of their terms can be included in their respective error terms.

2.3. Approximation of the Zaremba eigenvalue using the generalized argument principle. In this section we derive asymptotic expressions for the perturbation of the Zaremba eigenvalues when a small portion of the boundary is changed from Dirichlet to Neumann (see Figure 2.4).

Let $\Gamma_{\Delta} \subset \partial \Omega$ be a boundary interval of length $2 \varepsilon$. Let $\left(\Gamma_{D} \bullet \Gamma_{\Delta}, \Gamma_{\mathrm{N}}\right)$ be a partition of $\partial \Omega$. We associate the operator $\mathcal{A}_{0}(k)$, defined via (2.5), to that partition. This corresponds to $\Gamma_{\Delta}$ having a Dirichlet boundary condition. Then we define $\mathcal{A}_{\varepsilon}(k)$, also by obvious changes in the integrals in (2.5), to be the operator associated to the partition $\left(\Gamma_{\mathrm{D}}, \Gamma_{\mathrm{N}} \cup \Gamma_{\Delta}\right)$. This in turn corresponds to $\Gamma_{\Delta}$ being a Neumann part. For ease of notation, we define $k_{j}^{0}:=\sqrt{\lambda_{j}^{\Gamma_{\mathrm{D}} \cup \Gamma_{\Delta}}}$ and $k_{j}^{\varepsilon}:=\sqrt{\lambda_{j}^{\Gamma_{\mathrm{D}}}}$ for all $j \in \mathbb{N}$ and call those characteristic values to their respective operators. From [7, Lemma 3.8] we then have the following lemma.

Lemma 2.3. Let $k_{j}^{0}$ be a simple characteristic value. Let $V \subset \mathbb{C}$ be a neighborhood of $k_{j}^{0}$ such that $k_{j}^{\varepsilon} \in V$. Assume further that no other square root of the Zaremba eigenvalue to the partition $\left(\Gamma_{D}, \Gamma_{N} \cup \Gamma_{\Delta}\right)$ of $\partial \Omega$ is in $\bar{V}$. Then $k_{j}^{\varepsilon}$ is given by the contour integral

$$
k_{j}^{\varepsilon}-k_{j}^{0}=\frac{1}{2 \pi i} \operatorname{tr} \int_{\partial V}\left(\omega-k_{j}^{0}\right) \mathcal{A}_{\varepsilon}(\omega)^{-1} \partial_{\omega} \mathcal{A}_{\varepsilon}(\omega) \mathrm{d} \omega .
$$

Copyright $@$ by SIAM. Unauthorized reproduction of this article is prohibited. 


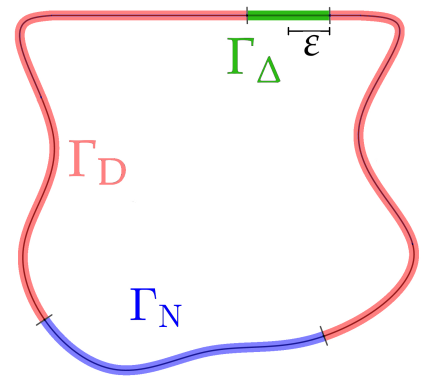

FIG. 2.4. An example for a domain with a Neumann boundary and a Dirichlet boundary and a small straight arc $\Gamma_{\Delta}$ of length $2 \varepsilon$. We associate $k_{j}^{0}$ with $\Gamma_{\Delta}$ being a Dirichlet boundary and $k_{j}^{\varepsilon}$ with $\Gamma_{\Delta}$ being a Neumann boundary.

Here $\partial_{\omega}$ denotes the variation of the operator in the wavenumber parameter $\omega$. This expression is exact. Unfortunately, its use in a practical algorithm is limited, since it would entail inverting the operator $\mathcal{A}_{\varepsilon}(\omega)$ for each $\varepsilon$ used in an optimization. It is useful, therefore, to locate an expression in which this inverse is approximated by $\mathcal{A}_{0}(\omega)$ instead.

From [7, Theorem 3.12] we get the approximation

$$
k_{j}^{\varepsilon}-k_{j}^{0} \approx \frac{-1}{2 \pi i} \operatorname{tr} \int_{\partial V} \mathcal{A}_{0}(\omega)^{-1}\left(\mathcal{A}_{\varepsilon}(\omega)-\mathcal{A}_{0}(\omega)\right) \mathrm{d} \omega,
$$

where we expect the error to be in $o\left(\frac{1}{|\log (\varepsilon)|}\right)$. We can, in fact, obtain a faster and even more accurate approximation, which we describe in the following proposition.

Proposition 2.4. Let $k_{j}^{0}$ be the $j$ th (sorted) characteristic value of $\mathcal{A}_{0}(k)$ corresponding to the decomposition $\Gamma_{D}, \Gamma_{N}$, and assume it is simple. Then one can find an $\varepsilon>0$ and a neighborhood $V \subset \mathbb{C}$ containing $k_{j}^{0}$ so that

- the jth characteristic value $k_{j}^{\varepsilon}$ of the operator $\mathcal{A}_{\varepsilon}(k)$ (obtained by changing $\Gamma_{\Delta}$ to a Neumann boundary condition) is contained $\in V$;

- no other square root of the Laplace eigenvalues to the partition $\left(\Gamma_{D}, \Gamma_{N} \bullet \Gamma_{\Delta}\right)$ of $\partial \Omega$ are in $\bar{V}$;

- the characteristic value of the perturbed operator $k_{j}^{\varepsilon}$ is given by

$$
\begin{aligned}
k_{j}^{\varepsilon}-k_{j}^{0}= & \frac{-1}{2 \pi i} \operatorname{tr} \int_{\partial V}\left(\mathrm{I}+\left(\omega-k_{j}^{0}\right) \mathcal{A}_{\varepsilon}\left(k_{j}^{0}\right)^{-1} \partial_{\omega} \mathcal{A}_{\varepsilon}\left(k_{j}^{0}\right)\right)^{-1} \mathrm{~d} \omega \\
& \times\left[1+\mathcal{O}\left(\operatorname{tr} \int_{\partial V}\left(\mathrm{I}+\left(\omega-k_{j}^{0}\right) \mathcal{A}_{\varepsilon}\left(k_{j}^{0}\right)^{-1} \partial_{\omega} \mathcal{A}_{\varepsilon}\left(k_{j}^{0}\right)\right)^{-1} \mathrm{~d} \omega\right)\right] .
\end{aligned}
$$

Here I is the identity operator.

Proof. We first observe from Proposition 2.1, together with the fact that $\mathcal{A}_{\varepsilon}(k)$ is a Fredholm analytic operator of index 0 in $\mathbb{C} \backslash i \mathbb{R}^{-}$, that we can see that $k_{j}^{\varepsilon} \nearrow k_{j}^{0}$ for $\varepsilon \searrow 0^{+}$. We now examine the perturbed operator $\mathcal{A}_{\varepsilon}$. Its characteristic value is $k_{j}^{\varepsilon}$. Provided $k_{j}^{0}$ is sufficiently close to $k_{j}^{\varepsilon}$, we have the following Taylor expansion:

$$
\mathcal{A}_{\varepsilon}(\omega)=\mathcal{A}_{\varepsilon}\left(k_{j}^{0}\right)+\left(\omega-k_{j}^{0}\right) \partial_{\omega} \mathcal{A}_{\varepsilon}\left(k_{j}^{0}\right)+\mathcal{B}_{\varepsilon}(\omega),
$$

where $\mathcal{B}_{\varepsilon}(\omega)=\mathcal{O}\left(\left(\omega-k_{j}^{0}\right)^{2}\right)$. This expansion holds only in a neighborhood $V_{\varepsilon}^{0}$ of $k_{j}^{0}$, and so $\varepsilon$ must be small enough such that $k_{j}^{0} \in V_{\varepsilon}^{0}$. 
Then consider that we have in the operator norm

$$
\left\|\left(\mathcal{A}_{\varepsilon}\left(k_{j}^{0}\right)+\left(\omega-k_{j}^{0}\right) \partial_{\omega} \mathcal{A}_{\varepsilon}\left(k_{j}^{0}\right)\right)^{-1} \mathcal{B}_{\varepsilon}(\omega)\right\|<1
$$

for $\omega \in V_{\varepsilon} \subset V_{\varepsilon}^{0}$ close enough to both $k_{j}^{\varepsilon}$ and $k_{j}^{0}$, because then the Taylor remainder $\mathcal{B}_{\varepsilon}(\omega)=\mathcal{O}\left(\left(\omega-k_{j}^{0}\right)^{2}\right)$. If $\varepsilon$ is small enough, then $k_{j}^{\varepsilon} \in V_{\varepsilon}$. Then by the generalization of Rouché's theorem [7, Theorem 1.15] we have that since $\mathcal{A}_{\varepsilon}\left(k_{j}^{0}\right)+\left(\omega-k_{j}^{0}\right) \partial_{\omega} \mathcal{A}_{\varepsilon}\left(k_{j}^{0}\right)$ and $\mathcal{A}_{\varepsilon}(\omega)$ are close in operator norm, they both have the same number of characteristic values in $V_{\varepsilon}$. Thus $\mathcal{A}_{\varepsilon}\left(k_{j}^{0}\right)+\left(\omega-k_{j}^{0}\right) \partial_{\omega} \mathcal{A}_{\varepsilon}\left(k_{j}^{0}\right)$ has a simple characteristic value $k_{j}^{\sharp}$ in $V_{\varepsilon}$. Now we can use Lemma 2.3 but replace $\mathcal{A}_{0}(\omega)$ by $\left(\mathcal{A}_{\varepsilon}\left(k_{j}^{0}\right)+\left(\omega-k_{j}^{0}\right) \partial_{\omega} \mathcal{A}_{\varepsilon}\left(k_{j}^{0}\right)\right)$ to get

$$
\begin{aligned}
k_{j}^{\sharp}-k_{j}^{0}= & \frac{1}{2 \pi i} \operatorname{tr} \int_{\partial V_{\varepsilon}}\left(\omega-k_{j}^{0}\right)\left(\mathcal{A}_{\varepsilon}\left(k_{j}^{0}\right)+\left(\omega-k_{j}^{0}\right) \partial_{\omega} \mathcal{A}_{\varepsilon}\left(k_{j}^{0}\right)\right)^{-1} \\
& \times \partial_{\omega}\left(\mathcal{A}_{\varepsilon}\left(k_{j}^{0}\right)+\left(\omega-k_{j}^{0}\right) \partial_{\omega} \mathcal{A}_{\varepsilon}\left(k_{j}^{0}\right)\right) \mathrm{d} \omega \\
= & \frac{1}{2 \pi i} \operatorname{tr} \int_{\partial V_{\varepsilon}}\left(\omega-k_{j}^{0}\right)\left(\mathcal{A}_{\varepsilon}\left(k_{j}^{0}\right)+\left(\omega-k_{j}^{0}\right) \partial_{\omega} \mathcal{A}_{\varepsilon}\left(k_{j}^{0}\right)\right)^{-1} \partial_{\omega} \mathcal{A}_{\varepsilon}\left(k_{j}^{0}\right) \mathrm{d} \omega,
\end{aligned}
$$

and hence,

$$
\begin{aligned}
k_{j}^{\sharp}-k_{j}^{0}= & \frac{1}{2 \pi i} \operatorname{tr} \int_{\partial V_{\varepsilon}}\left(\mathcal{A}_{\varepsilon}\left(k_{j}^{0}\right)+\left(\omega-k_{j}^{0}\right) \partial_{\omega} \mathcal{A}_{\varepsilon}\left(k_{j}^{0}\right)\right)^{-1} \\
& \times\left(\mathcal{A}_{\varepsilon}\left(k_{j}^{0}\right)+\left(\omega-k_{j}^{0}\right) \partial_{\omega} \mathcal{A}_{\varepsilon}\left(k_{j}^{0}\right)-\mathcal{A}_{\varepsilon}\left(k_{j}^{0}\right)\right) \mathrm{d} \omega \\
= & \frac{1}{2 \pi i} \operatorname{tr}\left(\int_{\partial V_{\varepsilon}} \mathrm{I} \mathrm{d} \omega-\int_{\partial V_{\varepsilon}}\left(\mathcal{A}_{\varepsilon}\left(k_{j}^{0}\right)+\left(\omega-k_{j}^{0}\right) \partial_{\omega} \mathcal{A}_{\varepsilon}\left(k_{j}^{0}\right)\right)^{-1} \mathcal{A}_{\varepsilon}\left(k_{j}^{0}\right) \mathrm{d} \omega\right) \\
= & -\frac{1}{2 \pi i} \operatorname{tr} \int_{\partial V_{\varepsilon}}\left(\mathcal{A}_{\varepsilon}\left(k_{j}^{0}\right)+\left(\omega-k_{j}^{0}\right) \partial_{\omega} \mathcal{A}_{\varepsilon}\left(k_{j}^{0}\right)\right)^{-1} \mathcal{A}_{\varepsilon}\left(k_{j}^{0}\right) \mathrm{d} \omega .
\end{aligned}
$$

Moreover, by a standard perturbation argument [7, section 5.2.4], we have at the leading-order term

$$
k_{j}^{\varepsilon}-k_{j}^{\sharp}=-\left(\mathcal{B}_{\varepsilon}\left(k_{j}^{\sharp}\right) \psi_{j}^{\sharp}, \psi_{j}^{\sharp}\right),
$$

where $\psi_{j}^{\sharp}$ is the root function associated with the characteristic value $k_{j}^{\sharp}$ evaluated at $k_{j}^{\sharp}$. Thus,

$$
k_{j}^{\varepsilon}-k_{j}^{0}=\left(k_{j}^{\sharp}-k_{j}^{0}\right)\left(1+\mathcal{O}\left(k_{j}^{\sharp}-k_{j}^{0}\right)\right),
$$

and therefore, Proposition 2.4 holds.

We remark on the significance of this result from the point of view of computation, and which makes it a key ingredient in our algorithm. If one seeks a high-accuracy approximation of the characteristic value $k_{j}^{\varepsilon}$ of $\mathcal{A}_{\varepsilon}(k)$ and one already has a good approximation of $k_{j}^{0}$, the approximation in Proposition 2.4 allows us to proceed by assembling only one matrix, that corresponding to $\mathcal{A}_{\varepsilon}\left(k_{j}^{0}\right)$. The contour integrals can be effectively computed using the trapezoidal rule, making this an inexpensive but very accurate approximation of $k_{j}^{\varepsilon}$. 
2.4. Approximation of the Zaremba function. Let $\Omega:=\{z \in \mathbb{C}|| z \mid<1\}$, and let $\Gamma_{\Delta} \subset \partial \Omega$ be a boundary interval of length $2 \varepsilon$ with center $y_{\star} \in \Gamma_{\Delta}$. Let $(\partial \Omega, \varnothing)$ be the partition of $\partial \Omega$, and with it we associate the Zaremba function $\mathrm{Z}_{\mathrm{D}}^{k}\left(x_{\mathrm{S}}, \cdot\right)$ for $x_{\mathrm{S}} \in \Omega$, defined via (2.1). This corresponds to $\Gamma_{\Delta}$ having a Dirichlet boundary condition. Then we introduce $\mathrm{Z}_{\mathrm{N}}^{k}\left(x_{\mathrm{S}}, \cdot\right) \in \mathrm{L}^{2}(\Omega), x_{\mathrm{S}} \in \Omega$, also defined via (2.1), to be the Zaremba function associated to the partition $\left(\partial \Omega \backslash \overline{\Gamma_{\Delta}}, \Gamma_{\Delta}\right)$. This in turn corresponds to $\Gamma_{\Delta}$ having a Neumann boundary condition. We then have the following lemma.

Lemma 2.5. Let $\Omega, y_{\star}, \Gamma_{\Delta}, \mathrm{Z}_{\mathrm{D}}^{k}\left(x_{S}, \cdot\right)$ and $\mathrm{Z}_{\mathrm{N}}^{k}\left(x_{S}, \cdot\right)$ be defined as described above. Let $\varepsilon>0$ be small enough. Let $k>0$ be such that $k^{2} \neq \lambda_{j}^{\partial \Omega \backslash \Gamma_{\Delta}}$ and $k^{2} \neq \lambda_{j}^{\partial \Omega}$ for all $j \in \mathbb{N}$. Then for all $z \in \Omega$,

$$
\mathrm{Z}_{\mathrm{N}}^{k}\left(x_{S}, z\right)=\mathrm{Z}_{\mathrm{D}}^{k}\left(x_{S}, z\right)-\varepsilon^{2} \frac{\pi}{2} \partial_{\nu_{y_{\star}}} \mathrm{Z}_{\mathrm{D}}^{k}\left(z, y_{\star}\right) \partial_{\nu_{y_{\star}}} \mathrm{Z}_{\mathrm{D}}^{k}\left(x_{S}, y_{\star}\right)+\mathcal{O}\left(\frac{\varepsilon^{2}}{|\log (\varepsilon / 2)|^{2}}\right) .
$$

Lemma 2.5 follows readily from combining the results in $[4$, Theorem 5.4$]$ and $[4$, equation (6.24)]

Numerical experiments confirm that $\left|\mathrm{Z}_{\mathrm{N}, x_{\mathrm{S}}}^{k}(y)-\mathrm{Z}_{\mathrm{D}, x_{\mathrm{S}}}^{k}(y)\right|$ is of order of $\varepsilon^{2}$, as long as $y$ is far enough away from the boundary.

3. Spectral decomposition of the Zaremba function. Let us again consider the more general setup at the beginning of section 2 ; that is, let $\left(\Gamma_{\mathrm{D}}, \Gamma_{\mathrm{N}}\right)$ be a partition of $\partial \Omega$, let $\left\{\lambda_{j}^{\Gamma_{\mathrm{D}}}\right\}_{j=1}^{\infty}$ be the Zaremba eigenvalues, and let $\left\{u_{j}\right\}_{j=1}^{\infty}$ be an $L^{2}$-orthonormal basis of associated eigenfunctions. Then we have the following statement about the Zaremba function $\mathrm{Z}_{x_{\mathrm{S}}}^{k}, x_{\mathrm{S}} \in \Omega$, defined by (2.1).

TheOREM 3.1. For all $y \in \Omega, y \neq x_{S}$ and for all $k>0$ which are not in the spectrum, i.e., $k^{2} \neq \lambda_{j}^{\Gamma_{D}}$ of the Zaremba eigenvalue problem, the Zaremba function $\mathrm{Z}_{x_{S}}^{k}$, given by $(2.1)$, exists and is in $\mathrm{L}_{\text {loc }}^{2}(\Omega)$. Furthermore, we can write it as

$$
\mathrm{Z}_{x_{S}}^{k}(y)=\sum_{j=1}^{\infty} \frac{u_{j}\left(x_{S}\right) u_{j}(y)}{k^{2}-\lambda_{j}^{\Gamma_{D}}}
$$

Next, we will consider the proof of Theorem 3.1. To this end, we define

$$
\mathrm{H}_{0, \Gamma_{\mathrm{D}}}^{1}(\Omega):=\left\{v \in \mathrm{H}^{1}(\Omega)|v|_{\Gamma_{\mathrm{D}}}=0\right\} .
$$

Consider that the solution to the Laplace eigenvalue equation $u \in \mathrm{H}_{0, \Gamma_{\mathrm{D}}}^{1}(\Omega)$. Let

$$
\operatorname{dom}(-\triangle):=\left\{w \in \mathrm{H}_{0, \Gamma_{\mathrm{D}}}^{1}(\Omega)\left|\triangle w \in \mathrm{L}^{2}(\Omega), \partial_{\nu} w\right|_{\Gamma_{\mathrm{N}}}=0\right\} .
$$

The operator $-\triangle$ is self-adjoint in $\mathrm{L}^{2}(\Omega)$, which we readily see using Green's identity, and it has thus a discrete spectrum. Moreover, $-\triangle$ corresponds to the sesquilinear form $\left\langle v_{1}, v_{2}\right\rangle \mapsto\left(\nabla v_{1}, \nabla v_{2}\right)_{\mathrm{L}^{2}(\Omega)}$ with domain $\mathrm{H}_{0, \Gamma_{\mathrm{D}}}^{1}$, since

$$
\left(-\triangle w_{1}, w_{2}\right)_{\mathrm{L}^{2}(\Omega)}=\left(\nabla w_{1}, \nabla w_{2}\right)_{\mathrm{L}^{2}(\Omega)}
$$

for all $w_{1}, w_{2} \in \operatorname{dom}(-\triangle)$; see $[8,14,20]$ for more details on semibounded selfadjoint operators and corresponding quadratic forms. And the form $\langle\cdot, \cdot\rangle$ is closed, nonnegative, and symmetric. This allows us to use the min-max principle. Thus we can write for all $j \in \mathbb{N}$,

$$
\lambda_{j}^{\Gamma_{\mathrm{D}}}=\min _{\substack{L \subset \mathrm{H}_{0, \Gamma \mathrm{D}}^{1}(\Omega) \\ \operatorname{dim} L=j}} \max _{v \in L \backslash\{0\}} \frac{\|\nabla v\|_{\mathrm{L}^{2}(\Omega)}^{2}}{\|v\|_{\mathrm{L}^{2}(\Omega)}^{2}} .
$$

This leads us to the following lemma. 
LEMma 3.2. For all $f \in \operatorname{dom}(-\triangle)$, we have that

$$
\left\|f-\sum_{j=1}^{N} c_{j} u_{j}\right\|_{L^{2}(\Omega)}^{2}=\int_{\Omega}\left|f-\sum_{j=1}^{N} c_{j} u_{j}\right|^{2} \mathrm{~d} x \stackrel{N \rightarrow \infty}{\longrightarrow} 0,
$$

where $c_{j}:=\left(f, u_{j}\right)_{\mathrm{L}^{2}(\Omega)}$, that is, the linear subset spanned by eigenfunctions of the Laplace eigenvalue equation with mixed boundary conditions (2.3) is dense in $\operatorname{dom}(-\triangle)$.

Proof. Let $\mathrm{r}_{N}:=f-\sum_{j=1}^{N} c_{j} u_{j}$. Then for all $i=1, \ldots, N$, we have that

$$
\begin{aligned}
\left(\mathrm{r}_{N}, u_{i}\right)_{\mathrm{L}^{2}(\Omega)} & =\left(f-\sum_{j=1}^{N} c_{j} u_{j}, u_{i}\right)_{\mathrm{L}^{2}}=\left(f, u_{i}\right)_{\mathrm{L}^{2}}-c_{i}\left(u_{i}, u_{j}\right)_{\mathrm{L}^{2}}=0, \\
\left(\nabla \mathrm{r}_{N}, \nabla u_{i}\right)_{\mathrm{L}^{2}(\Omega)} & =\left(\nabla f, \nabla u_{i}\right)_{\mathrm{L}^{2}}-\sum_{j=1}^{N} c_{j}\left(\nabla u_{j}, \nabla u_{i}\right)_{\mathrm{L}^{2}} \\
& =\lambda_{i}^{\Gamma_{\mathrm{D}}}\left(f, u_{i}\right)_{\mathrm{L}^{2}}-\lambda_{i}^{\Gamma_{\mathrm{D}}} c_{j}\left(u_{i}, u_{i}\right)_{\mathrm{L}^{2}}=0,
\end{aligned}
$$

where we used Green's identity and the fact that $f, u_{j} \in \operatorname{dom}(-\triangle)$. Next, we want to show that

$$
\lambda_{N}^{\Gamma_{\mathrm{D}}} \leq \frac{\left\|\nabla \mathrm{r}_{N}\right\|_{\mathrm{L}^{2}(\Omega)}^{2}}{\left\|\mathrm{r}_{N}\right\|_{\mathrm{L}^{2}(\Omega)}^{2}} .
$$

To this end, applying the min-max principle (3.1) yields

$$
\begin{aligned}
\lambda_{j}^{\Gamma_{\mathrm{D}}} & \leq \max _{v \in \operatorname{span}\left\{u_{1}, \ldots, u_{N-1}, \mathrm{r}_{N}\right\}} \frac{\|\nabla v\|_{\mathrm{L}^{2}(\Omega)}^{2}}{\|v\|_{\mathrm{L}^{2}(\Omega)}^{2}} \\
& =\max _{a_{1}, \ldots, a_{N} \in \mathbb{R}} \frac{\left\|\nabla\left(a_{N} \mathrm{r}_{N}+a_{1} v_{1}+\cdots+a_{n-1} v_{n-1}\right)\right\|^{2}}{\left\|a_{N} \mathrm{r}_{N}+a_{1} v_{1}+\cdots a_{n-1} v_{n-1}\right\|^{2}} \\
& =\max _{a_{1}, \ldots, a_{N} \in \mathbb{R}} \frac{a_{N}^{2}\left\|\nabla \mathrm{r}_{N}\right\|^{2}+a_{1}^{2}\left\|\nabla v_{1}\right\|^{2}+\cdots+a_{n-1}^{2}\left\|\nabla v_{n-1}\right\|^{2}}{a_{N}^{2}\left\|\mathrm{r}_{N}\right\|^{2}+a_{1}^{2}\left\|v_{1}\right\|^{2}+\cdots+a_{n-1}^{2}\left\|v_{n-1}\right\|^{2}} \\
& =\max _{a_{1}, \ldots, a_{N} \in \mathbb{R}} \frac{a_{N}^{2}\left\|\nabla \mathrm{r}_{N}\right\|^{2}+\lambda_{1}^{\Gamma_{\mathrm{D}}} a_{1}^{2}+\cdots+\lambda_{n-1}^{\Gamma_{\mathrm{D}}} a_{n-1}^{2}}{a_{N}^{2}\left\|\mathrm{r}_{N}\right\|^{2}+a_{1}^{2}+\cdots+a_{n-1}^{2}} \\
& \leq \max _{a_{1}, \ldots, a_{N} \in \mathbb{R}} \frac{a_{N}^{2}\left\|\nabla \mathrm{r}_{N}\right\|^{2}+\lambda_{n-1}^{\Gamma_{\mathrm{D}}}\left(a_{1}^{2}+\cdots+a_{n-1}^{2}\right)}{a_{N}^{2}\left\|\mathrm{r}_{N}\right\|^{2}+a_{1}^{2}+\cdots+a_{n-1}^{2}}
\end{aligned}
$$

Thus, we can infer $\lambda_{N}^{\Gamma_{\mathrm{D}}} \leq \frac{\left\|\nabla \mathrm{r}_{N}\right\|^{2}}{\left\|\mathrm{r}_{N}\right\|^{2}}$ from $\lambda_{n-1}^{\Gamma_{\mathrm{D}}} \leq \frac{\left\|\nabla \mathrm{r}_{N}\right\|^{2}}{\left\|\mathrm{r}_{N}\right\|^{2}}$, which in turn is given by an induction argument, whose induction basis follows trivially from the min-max principle (3.1). Using the definition of $c_{j}$, we have that

$$
\begin{aligned}
\left\|\nabla \mathrm{r}_{N}\right\|_{\mathrm{L}^{2}(\Omega)}^{2} & =\|\nabla f\|_{\mathrm{L}^{2}(\Omega)}^{2}-2 \sum_{j=1}^{N} c_{j} \lambda_{j}^{\Gamma_{\mathrm{D}}}\left(f, u_{j}\right)_{\mathrm{L}^{2}(\Omega)}+\sum_{j=1}^{N} c_{j}^{2} \lambda_{j}^{\Gamma_{\mathrm{D}}}\left\|u_{j}\right\|_{\mathrm{L}^{2}(\Omega)}^{2} \\
& =\|\nabla f\|_{\mathrm{L}^{2}(\Omega)}^{2}-\sum_{j=1}^{N} \lambda_{j}^{\Gamma_{\mathrm{D}}}\left(f, u_{j}\right)_{\mathrm{L}^{2}(\Omega)}^{2} \\
& \leq\|\nabla f\|_{\mathrm{L}^{2}(\Omega)}^{2} .
\end{aligned}
$$

Copyright (c) by SIAM. Unauthorized reproduction of this article is prohibited. 
Thus, using (3.3), we have that

$$
\left\|\mathrm{r}_{N}\right\|_{\mathrm{L}^{2}}^{2} \leq \frac{\|\nabla f\|_{\mathrm{L}^{2}}^{2}}{\lambda_{N}^{\Gamma_{\mathrm{D}}}}
$$

Since $\|\nabla f\|_{\mathrm{L}^{2}}^{2}=(f,-\triangle f)_{\mathrm{L}^{2}} \leq\|f\|_{\mathrm{L}^{2}}\|\triangle f\|_{\mathrm{L}^{2}}<\infty,\|\nabla f\|_{\mathrm{L}^{2}}^{2}$ is bounded. Using the fact that $\lambda_{N}^{\Gamma_{\mathrm{D}}} \stackrel{N \rightarrow \infty}{\longrightarrow} \infty$, we have that $\left\|\mathrm{r}_{N}\right\|_{\mathrm{L}^{2}}^{2} \stackrel{N \rightarrow \infty}{\longrightarrow} 0$. This completes the proof of Lemma 3.2 .

Proof of Theorem 3.1. To show the existence of the Zaremba function $\mathrm{Z}_{x_{\mathrm{S}}}^{k}$, we write $\mathrm{Z}_{x_{\mathrm{S}}}^{k}(y)$ for all $y \in \Omega, y \neq x_{\mathrm{S}}$ as

$$
\mathrm{Z}_{x_{\mathrm{S}}}^{k}(y)=\Gamma^{k}\left(x_{\mathrm{S}}, y\right)+\mathrm{R}^{k}\left(x_{\mathrm{S}}, y\right),
$$

where $\Gamma^{k}$ is the fundamental solution to the Helmholtz equation and $\mathrm{R}^{k}$ satisfies

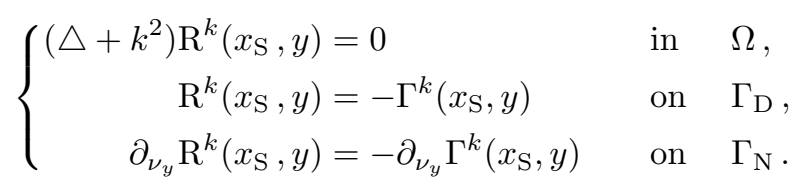

The solution to (3.6) does exist for those values of $k$ specified in the theorem, and it is in $\mathrm{H}^{1}(\Omega)$; see [17, Theorem 4.10]. Using that $\Gamma^{k}\left(x_{\mathrm{S}}, \cdot\right) \in L^{2}(\Omega)$, we have that $\mathrm{Z}_{x \mathrm{~S}}^{k}(y) \in L^{2}(\Omega)$. Thus from Lemma 3.2 and the density of $\operatorname{dom}(-\triangle)$ in $L^{2}(\Omega)$, we have that for all $y \in \Omega, y \neq x_{\mathrm{S}}$,

$$
\mathrm{Z}_{x_{\mathrm{S}}}^{k}(y)=\sum_{j=1}^{\infty} a_{j} u_{j}(y)
$$

for some $a_{j} \in \mathbb{R}$, depending on $x_{\mathrm{S}}$. Let us give an expression for the $a_{j}$. Using Green's identity, we have that

$$
\begin{aligned}
u_{i}\left(x_{\mathrm{S}}\right) & =\int_{\Omega}\left(\triangle+k^{2}\right) \mathrm{Z}_{x_{\mathrm{S}}}^{k}(y) u_{i}(y) \mathrm{d} y=\int_{\Omega} \mathrm{Z}_{x_{\mathrm{S}}}^{k}(y)\left(\triangle+k^{2}\right) u_{i}(y) \mathrm{d} y \\
& =\left(k^{2}-\lambda_{i}^{\Gamma_{\mathrm{D}}}\right) \int_{\Omega} \mathrm{Z}_{x_{\mathrm{S}}}^{k}(y) u_{i}(y) \mathrm{d} y=\left(k^{2}-\lambda_{i}^{\Gamma_{\mathrm{D}}}\right) \int_{\Omega} \sum_{j=1}^{\infty} a_{j} u_{j}(y) u_{i}(y) \mathrm{d} y \\
& =\left(k^{2}-\lambda_{i}^{\Gamma_{\mathrm{D}}}\right) \sum_{j=1}^{\infty} a_{j} \delta_{0}(i-j)=\left(k^{2}-\lambda_{i}^{\Gamma_{\mathrm{D}}}\right) a_{i}
\end{aligned}
$$

where we used Fubini's theorem to interchange summation and integration. With that we infer that for all $i \in \mathbb{N}$,

$$
a_{i}=\frac{u_{i}\left(x_{\mathrm{S}}\right)}{k^{2}-\lambda_{i}^{\Gamma_{\mathrm{D}}}}
$$

and this concludes the proof.

4. The algorithm. We next present our main algorithm for wave enhancement. We begin with a domain $\Omega$, the source point $x_{\mathrm{S}}$ and the receiver point $y$, both in $\Omega$, and a predetermined target value $k_{\star}$ corresponding to a desired transmission frequency.

Copyright $@$ by SIAM. Unauthorized reproduction of this article is prohibited. 
First, we determine the next higher Dirichlet eigenvalue to $k_{\star}^{2}$, which is done using a discretized version of the operator $\mathcal{A}(k)$ given in section 2. The discretization follows the procedure developed in [2].

Second, we determine a location $y_{\star}$ on the boundary $\partial \Omega$, which yields a higher absolute value of $\left|\mathrm{Z}_{x_{\mathrm{S}}}^{k}\left(x_{\mathrm{S}}, y\right)\right|$, when we insert a small enough Neumann boundary at that location. Finding the location is established using Lemma 2.5, that is, we find the local maxima or minima of

$$
\partial_{\nu_{\star}} \mathrm{Z}_{\mathrm{D}}^{k_{\star}}\left(x_{\mathrm{S}}, y_{\star}\right) \cdot \partial_{\nu_{y_{\star}}} \mathrm{Z}_{\mathrm{D}}^{k_{\star}}\left(y, y_{\star}\right) .
$$

The computation of the Zaremba function is done by solving the problem (2.2) using the procedure described in [1].

Third, we successively increase the Neumann boundary until the characteristic value hits the target characteristic value. The computation of the new characteristic value after a small increase of the Neumann boundary is achieved using Proposition 2.4. It might be that we need to increase the boundary initially by a large amount, and the resulting characteristic value has to be computed with the time-expensive procedure described in [2].

A more detailed explanation is given in the comments after Algorithm 1. We note here that changing a boundary part from the Dirichlet boundary condition to the Neumann one, the associated Laplace eigenvalue $\lambda_{j}^{\Gamma_{\mathrm{D}}}$ decreases, according to Proposition 2.1, and thus the characteristic value $\sqrt{\lambda_{j}^{\Gamma_{\mathrm{D}}}}$ decreases as well. Moreover, $\lambda_{j}^{\Gamma_{\mathrm{D}}}$ is between the Neumann and the Dirichlet eigenvalue; that is, $\lambda_{j}^{\varnothing} \leq \lambda_{j}^{\Gamma_{\mathrm{D}}} \leq \lambda_{j}^{\partial \Omega}$. Increasing boundary length enough, we eventually hit the target characteristic value $k_{\star}$, because $\cup_{j=1}^{\infty}\left(\lambda_{j}^{\varnothing}, \lambda_{j}^{\partial \Omega}\right)=(0, \infty)$, since $\lambda_{j+1}^{\varnothing}<\lambda_{j}^{\partial \Omega}$, as proved in [10].

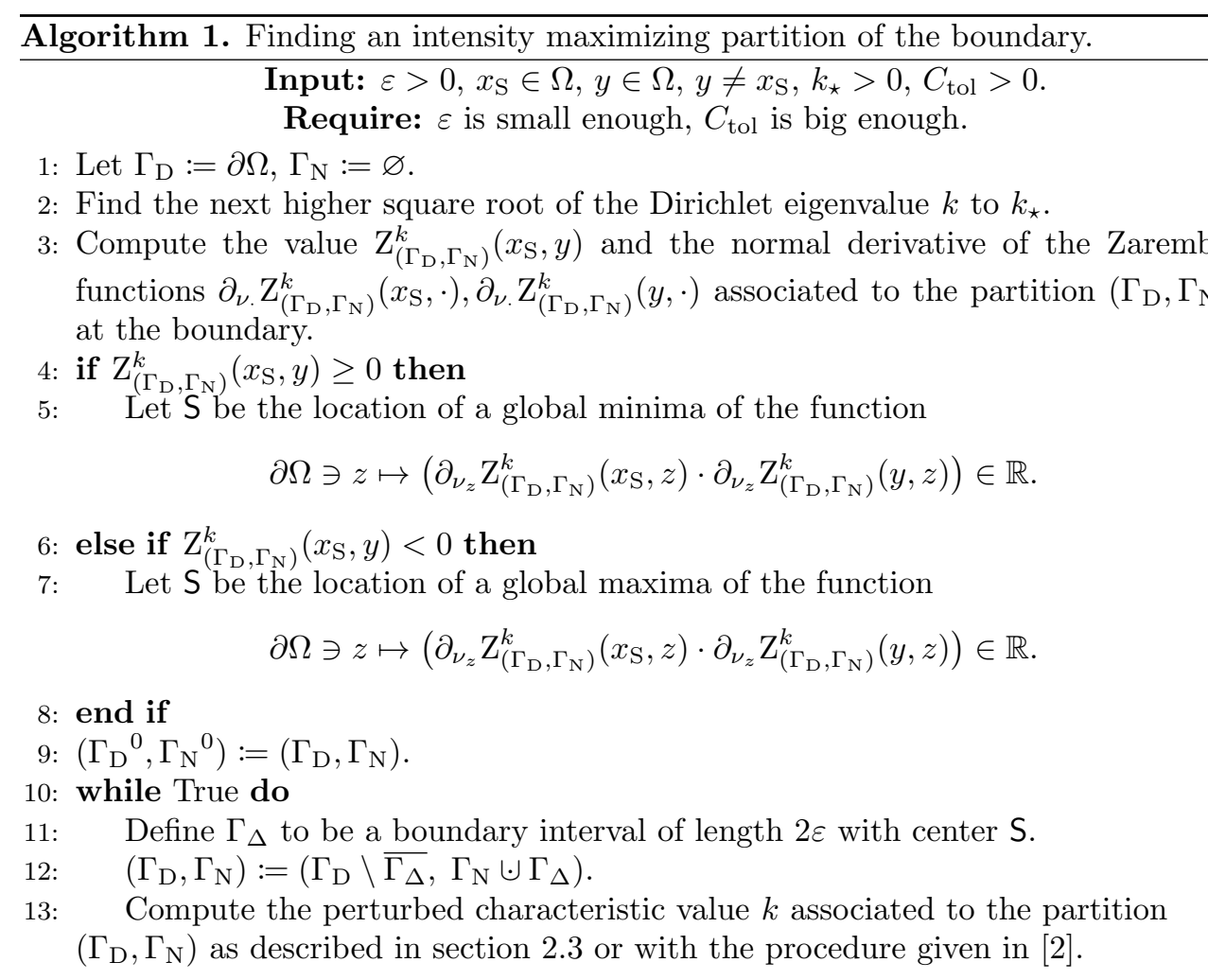




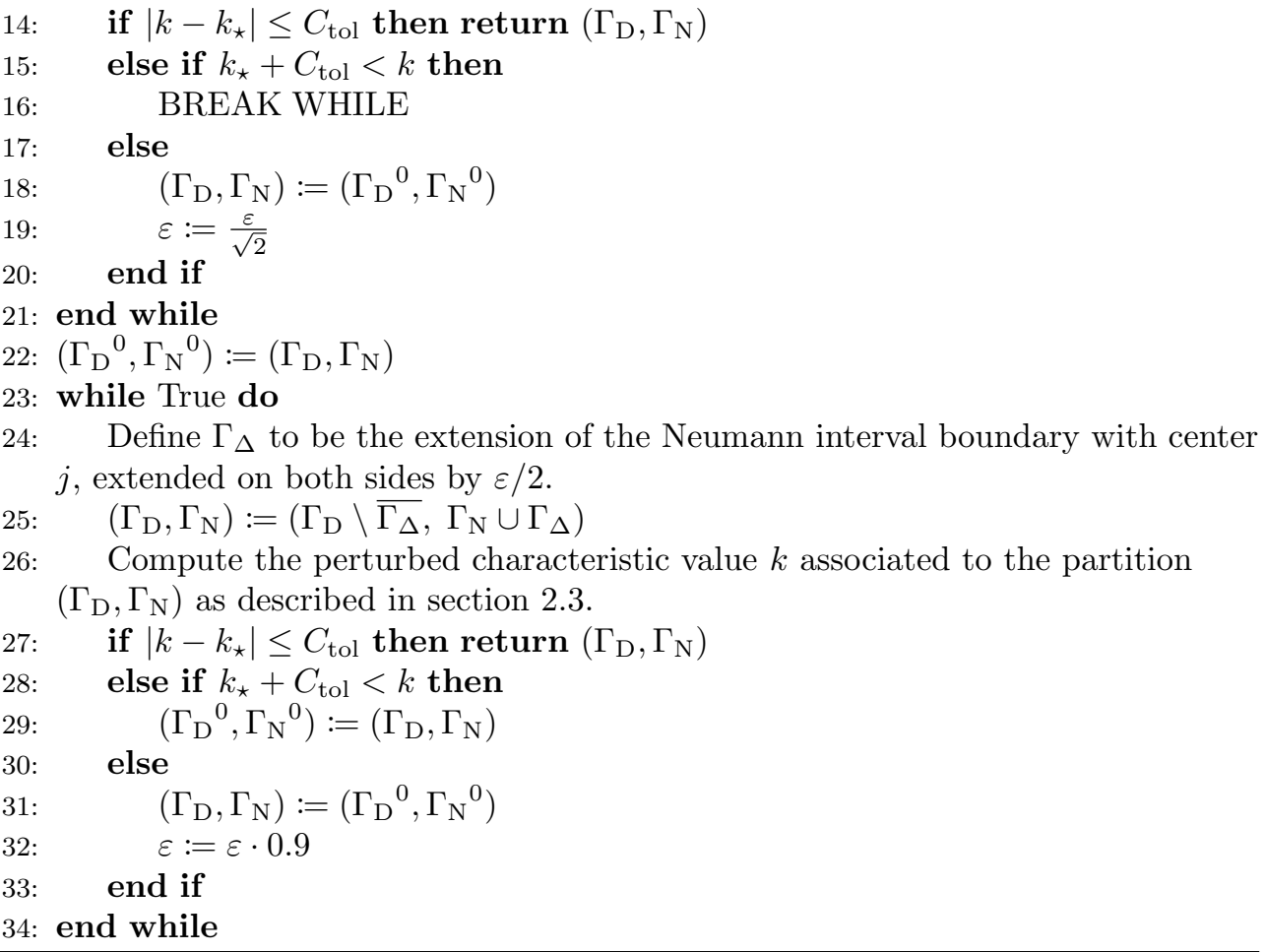

In the following we give an explanation for the choices.

Line 2: The reason we search for the next higher Dirichlet eigenvalue originates from the fact that, according to Proposition 2.1, when we insert Neumann boundaries, the corresponding eigenvalue decreases. The search for the next higher Dirichlet characteristic value and its multiplicity might be computationally expensive.

Line 3: Using the algorithm proposed in [1], we compute the Zaremba function using the decomposition $\mathrm{Z}^{k}\left(x_{\mathrm{S}}, y\right)=\Gamma^{k}\left(x_{\mathrm{S}}, y\right)+\mathrm{R}^{k}\left(x_{\mathrm{S}}, y\right)$, where $\Gamma^{k}$ is the fundamental solution to the Helmholtz equation and $\mathrm{R}^{k}$ satisfies (3.6). More exactly, we obtain a function $\varphi_{\mathrm{R}}$ on $\partial \Omega$, which is of the form in Proposition 2.2, with

$$
\mathrm{R}^{k}(y)=\int_{\partial \Omega} \Gamma^{k}(y, z) \varphi_{\mathrm{R}}(z) \mathrm{d} \sigma_{z}
$$

for $y \in \Omega$. Using the jump relations (see [7, section 2.3.2]), we get for $y \rightarrow \Gamma_{\mathrm{D}}$ that

$$
\partial_{\nu_{y}} \mathrm{R}^{k}(y)=\left(-\frac{1}{2} \mathrm{I}_{\partial \Omega}+\left(\mathcal{K}_{\partial \Omega}^{k}\right)^{*}\right)\left[\varphi_{\mathrm{R}}\right](y)
$$

where $\mathrm{I}_{\partial \Omega}$ denotes the identity operator.

Using a discretization to the operator $\left(\mathcal{K}_{\partial \Omega}^{k}\right)^{*}$, which we also readily obtain from [2], we can calculate $\partial_{\nu_{y}} \mathrm{Z}^{k}\left(x_{\mathrm{S}}, \cdot\right)=\partial_{\nu_{y}} \Gamma^{k}\left(x_{\mathrm{S}}, \cdot\right)+\partial_{\nu_{y}} \mathrm{R}^{k}\left(x_{\mathrm{S}}, \cdot\right)$.

Lines 4-8: In view of Lemma 2.5, we obtain that if $\mathrm{Z}_{\left(\Gamma_{\mathrm{D}}, \Gamma_{\mathrm{N}}\right)}^{k}\left(x_{\mathrm{S}}, y\right) \geq 0$, then we need a negative value of

$$
\partial_{\nu_{z}} \mathrm{Z}_{\left(\Gamma_{\mathrm{D}}, \Gamma_{\mathrm{N}}\right)}^{k}\left(x_{\mathrm{S}}, z\right) \cdot \partial_{\nu_{z}} \mathrm{Z}_{\left(\Gamma_{\mathrm{D}}, \Gamma_{\mathrm{N}}\right)}^{k}(y, z)
$$


to increase $\mathrm{Z}_{\left(\Gamma_{\mathrm{D}}, \Gamma_{\mathrm{N}}\right)}^{k}\left(x_{\mathrm{S}}, y\right)$ and vice versa for $\mathrm{Z}_{\left(\Gamma_{\mathrm{D}}, \Gamma_{\mathrm{N}}\right)}^{k}\left(x_{\mathrm{S}}, y\right) \leq 0$. Taking the minima, respectively, the maxima, we increase the absolute value of $\mathrm{Z}_{\left(\Gamma_{\mathrm{D}}, \Gamma_{\mathrm{N}}\right)}^{k}\left(x_{\mathrm{S}}, y\right)$.

We note that Lemma 2.5 only holds for the case where $\Omega$ is the unit circle, but we assume that it holds for all domains with smooth boundaries. We think that this can be established expanding the operator in $[4$, Theorem 5.4].

From Theorem 3.1 we know that the Zaremba function is real-valued, but due to numerical cancellation errors, the Zaremba function might have a nonzero imaginary part.

In our numerical experiments, it always holds that a global minima are negative and a global maxima are positive, respectively. But we do not know if this holds true in general.

Line 10: In this while-loop we change a boundary interval with center $\mathrm{S}$ and length $2 \varepsilon$ into a Neumann boundary condition. Then we compute an approximation $k$ to the new characteristic value. If $\left|k-k_{\star}\right|<C_{\text {tol }}$, we end the algorithm; if $k+C_{\mathrm{tol}}<k_{\star}$, we break the while-loop; and in the remaining case we decrease $\varepsilon$ and go through the loop again.

Line 13: To compute an approximation to the new characteristic value, which is smaller than $k$, we use the approximation stated in Proposition 2.4. To this end, we use as the complex domain $V$ encircling $k$ and $k_{\star}$ an ellipse with center $\left(k+k_{\star}\right) / 2$ and semimajor axis $\left(k-k_{\star}\right) \cdot 0.55$ and semiminor axis $\left(k-k_{\star}\right) \cdot 0.1$, which is to avoid complex characteristic values. A discretization to the operator $\mathcal{A}(k)$ is computed using the algorithm described in [2]. For the complex derivative of $\mathcal{A}(k)$, we used the rough approximation $(\mathcal{A}(w+0.01)-\mathcal{A}(w)) / 0.01$. The integral is approximated with a inbuilt process. The approximation may yield the same result as the former characteristic value, that is, $k$. In that case, the new characteristic value is not within $V$, which happens when the new boundary interval with Neumann boundary conditions is too long or cannot be detected by the approximation.

Here it might very well be that $k$ is not a simple eigenvalue but instead, for example, a double eigenvalue, which occurs for $\Omega$ being the unit circle. Then we search for both new eigenvalues and pick the one closer to $k_{\star}$ but still larger than $k_{\star}$. This search costs more time than the approximation algorithm.

In numerical experiments it seems that the two eigenvalues of the double Dirichlet eigenvalue split such that one eigenvalue escapes subjectively faster from the double Dirichlet eigenvalue the longer the new boundary interval $\Gamma_{\Delta}$ is and the other eigenvalue subjectively slower. This is reminiscent of the behavior of the perturbation of a double eigenvalue in [9], where the eigenvalue splits in an eigenvalue with difference $\mathcal{O}\left(\varepsilon^{2}\right)$ and an eigenvalue with difference $\mathcal{O}(1 /|\log (\varepsilon)|)$, where $\varepsilon$ is a value associated to the perturbation.

Line 23: Next, we expand the boundary interval, which we established in lines 1021. We expand it on both ends by a length $\varepsilon / 2$, whose factor $1 / 2$ is again chosen due to good numerical approval for minimizing runtime. Then we compute an approximation $k$ to the new characteristic value. If $\left|k-k_{\star}\right|<$ $C_{\text {tol }}$, we end the algorithm; if $k+C_{\text {tol }}<k_{\star}$, we extend the boundary interval once again; else decrease $\varepsilon$. 
Line 26: To compute an approximation to the new characteristic value, we use the same setting as in line 13: The complex domain $V$ encircling $k$ and $k_{\star}$ is an ellipse with center $\left(k+k_{\star}\right) / 2$ and semimajor axis $\left(k-k_{\star}\right) \cdot 0.55$ and semiminor axis $\left(k-k_{\star}\right) \cdot 0.1$. A discretization to the operator $\mathcal{A}(k)$ is computed using the algorithm described in [2]. For the complex derivative of said operator we used the rough approximation $(\mathcal{A}(w+0.01)-\mathcal{A}(w)) / 0.01$. The integral is approximated with a inbuilt process.

The approximation may again yield the same result as the former characteristic value, that is, $k$; this happens when $\Gamma_{\Delta}$ is too long.

In this while-loop, it never happened that $k$ is not a simple eigenvalue.

Remark 4.1. If the function $\partial \Omega \ni z \mapsto\left(\partial_{\nu_{z}} \mathrm{Z}_{\left(\Gamma_{D}, \Gamma_{N}\right)}^{k}\left(x_{\mathrm{S}}, z\right) \cdot \partial_{\nu_{z}} \mathrm{Z}_{\left(\Gamma_{D}, \Gamma_{N}\right)}^{k}(y, z)\right) \in$ $\mathbb{R}$ oscillates strongly on the boundary it might yield better results when multiple, but smaller, boundary intervals are applied. The thought behind this is that using one long boundary interval might intersect the disadvantageous part of the function $\partial_{\nu_{z}} \mathrm{Z}_{\left(\Gamma_{D}, \Gamma_{N}\right)}^{k}\left(x_{S}, z\right) \cdot \partial_{\nu_{z}} \mathrm{Z}_{\left(\Gamma_{D}, \Gamma_{N}\right)}^{k}(y, z)$ and thus decrease the intensity of $\mathrm{Z}_{\left(\Gamma_{D}, \Gamma_{N}\right)}^{k}\left(x_{S}, y\right)$.

5. Numerical implementation and tests. Our first numerical test shows the algorithm in the best case scenario. We have the domain $\Omega=\left\{x \in \mathbb{R}^{2} \mid\|x\|_{\mathbb{R}^{2}}<1\right\}$, the signal point $x_{\mathrm{S}}=(0,0)^{\mathrm{T}}$, the target characteristic value $k_{\star}=1$ and $C_{\mathrm{tol}}=10^{-3}$ and $\varepsilon=0.1$. We remark here that the next higher Dirichlet characteristic value is a simple one at approximately 2.40482. We let the receiving point $y \in\left\{(0, r)^{\mathrm{T}} \in\right.$ $\left.\mathbb{R}^{2} \mid r>0\right\}$ vary. Here we want to mention that our implementation of the Zaremba function, as described in section 4, comment on line 3, yields a nonzero imaginary part for the Zaremba function; the same holds true for the approximation to the characteristic value $k$ as described in section 4 , comment on line 13. We always choose the real part whenever in question. The number of discretization points for the operator $\mathcal{A}(k)$ was $3 \cdot 64$. The results are displayed in Table 5.1. The Zaremba functions with Dirichlet boundary conditions and with final mixed boundary conditions, for the case $y=(0,0.5)^{\mathrm{T}}$, are displayed in Figure 5.1.

Our second numerical test shows the algorithm for a higher target characteristic value $k_{\star}$, namely, $k_{\star}=15.4$. We have as the domain $\Omega$ the unit circle $\left\{x \in \mathbb{R}^{2} \mid\right.$ $\left.\|x\|_{\mathbb{R}^{2}}<1\right\}$, as the signal point $x_{\mathrm{S}}=(0,0)^{\mathrm{T}}$ and $C_{\text {tol }}=10^{-3}$ and $\varepsilon=0.05$. We remark here that the next higher Dirichlet characteristic value has multiplicity two and is at approximately 15.5898. We let the receiving point $y \in\left\{(0, r)^{\mathrm{T}} \in \mathbb{R}^{2} \mid r>0\right\}$ vary. The number of discretization points for the operator $\mathcal{A}(k)$ is $4 \cdot 48$. The results are displayed in Table 5.2. The Zaremba functions with Dirichlet boundary conditions

TABLE 5.1

We see Algorithm 1 performing on the unit circle with $k_{\star}=1, x_{S}=(0,0)^{\mathrm{T}}, y \in\left\{(0, r)^{\mathrm{T}} \in\right.$ $\left.\mathbb{R}^{2} \mid r>0\right\}, C_{\mathrm{tol}}=10^{-3}$, and $\varepsilon=0.1$. $\mathrm{Z}_{\text {Dirichlet }}^{k}\left(x_{S}, y\right)$ represents the Zaremba function on the partition $(\partial \Omega, \varnothing)$ of the boundary, and $\mathrm{Z}_{\mathrm{End}}^{k}\left(x_{S}, y\right)$ represents the Zaremba function on the final partition, where the final partition is made out of two boundary intervals, one with Dirichlet boundary conditions and the other with Neumann boundary conditions. $\theta_{\text {center }} \in[0,2 \cdot p i)$ represents the angle of the center of the Neumann boundary intervals and $l_{\mathrm{N}}$ its length. The shown values are the real, rounded values of the numerical results.

\begin{tabular}{rrrrrr} 
& $r=0.1$ & $r=0.25$ & $r=0.5$ & $r=0.75$ & $r=0.9$ \\
\hline $\mathrm{Z}_{\text {Dirichlet }}^{k_{\star}}\left(x_{\mathrm{S}}, y\right)$ & -0.412 & -0.261 & -0.138 & -0.059 & -0.022 \\
$\mathrm{Z}_{\text {End }}^{k_{\star}}\left(x_{\mathrm{S}}, y\right)$ & -1288 & -1438 & -1634 & -1754 & -1788 \\
$\mid \frac{\mathrm{Z}_{\text {End }}^{k_{\star}\left(x_{\mathrm{S}}, y\right)}}{\mathrm{Z}_{\text {Dirichlet }}^{k_{\star}\left(x_{\mathrm{S}}, y\right)} \mid}$ & 3123 & 5503 & 11824 & 29623 & 81687 \\
$\theta_{\text {center }}$ & $0.50 \pi$ & $0.50 \pi$ & $0.50 \pi$ & $0.50 \pi$ & $0.50 \pi$ \\
$l_{\mathrm{N}}$ & $1.32 \pi$ & $1.32 \pi$ & $1.32 \pi$ & $1.32 \pi$ & $1.32 \pi$ \\
\hline
\end{tabular}

Copyright $@$ by SIAM. Unauthorized reproduction of this article is prohibited. 

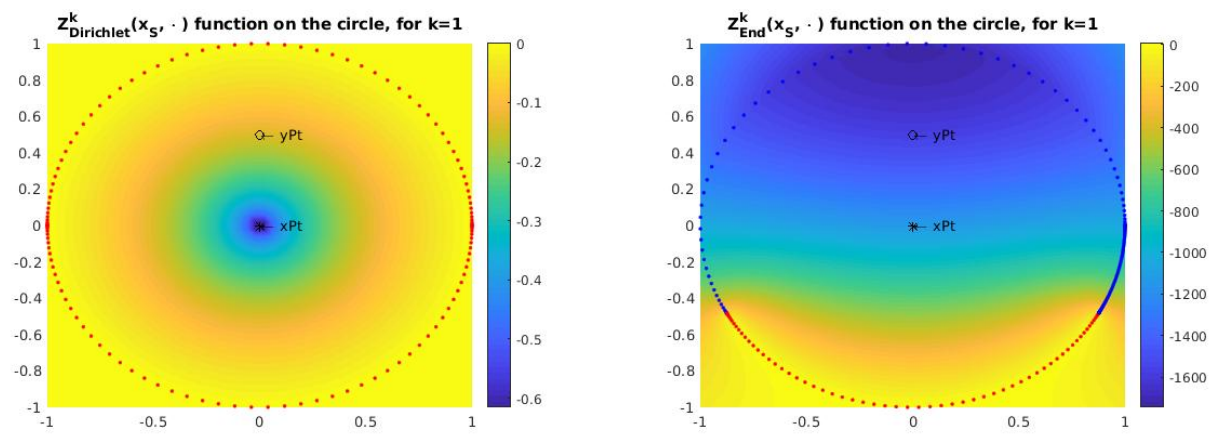

FIG. 5.1. The Zaremba function for $k_{\star}=1$ on the unit disk with Dirichlet boundary condition on the left and final mixed boundary conditions on the right. Marked are $x_{S}$, denoted as $x P t$, and $y$, denoted as yPt. The points on the boundary are our discretization points. Blue points denote the Neumann boundary conditions; red points denote the Dirichlet boundary conditions.

TABLE 5.2

We see Algorithm 1 performing on the unit circle with $k_{\star}=15.4, x_{S}=(0,0)^{\mathrm{T}}, y \in\left\{(0, r)^{\mathrm{T}} \in\right.$ $\left.\mathbb{R}^{2} \mid r>0\right\}, C_{\mathrm{tol}}=10^{-3}$, and $\varepsilon=0.05$. $\mathrm{Z}_{\text {Dirichlet }}^{k}\left(x_{S}, y\right), \mathrm{Z}_{\text {End }}^{k}\left(x_{S}, y\right), \theta_{\text {center }}$, and $l_{\mathrm{N}}$ are defined as in Table 5.1. The shown values are the real, rounded values of the numerical results.

\begin{tabular}{rrrrrr} 
& $r=0.1$ & $r=0.25$ & $r=0.5$ & $r=0.75$ & $r=0.9$ \\
\hline $\mathrm{Z}_{\text {Dirichlet }}^{k_{\star}}\left(x_{\mathrm{S}}, y\right)$ & 0.341 & -0.188 & 0.157 & -0.085 & 0.118 \\
$\mathrm{Z}_{\text {End }}^{k_{\star}}\left(x_{\mathrm{S}}, y\right)$ & 36.341 & -14.271 & 116.08 & -15.811 & 232.28 \\
$\left|\frac{\mathrm{z}_{\text {End }}^{k_{\star}}\left(x_{\mathrm{S}}, y\right)}{\mathrm{z}_{\text {Dirichlet }}^{k_{\star}}\left(x_{\mathrm{S}}, y\right)}\right|$ & 106.6 & 76.09 & 739.0 & 186.8 & 1962 \\
$\theta_{\text {center }}$ & $0.50 \pi$ & $1.90 \pi$ & $0.50 \pi$ & $0.46 \pi$ & $0.50 \pi$ \\
$l_{\mathrm{N}}$ & $0.064 \pi$ & $0.064 \pi$ & $0.064 \pi$ & $0.064 \pi$ & $0.064 \pi$ \\
\hline
\end{tabular}
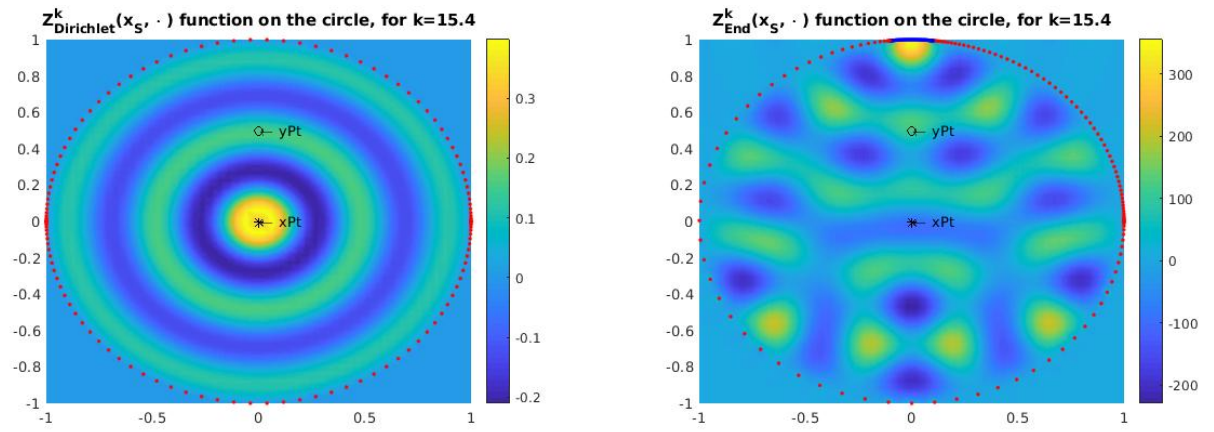

FIG. 5.2. The Zaremba function for $k_{\star}=15.4$ on the unit disk with Dirichlet boundary condition on the left and final mixed boundary conditions on the right. Further notation is as in Figure 5.1.

and with final mixed boundary conditions, for the case $y=(0,0.5)^{\mathrm{T}}$, are displayed in Figure 5.2.

Our third numerical test shows the algorithm for a different domain $\Omega$, namely, a kite-shaped domain given by the following description for its boundary:

$$
\left[\begin{array}{c}
\cos (\tau)+0.65 \cdot \cos (2 \cdot \tau)-0.65 \\
1.5 \cdot \sin (\tau)
\end{array}\right]
$$

Copyright (c) by SIAM. Unauthorized reproduction of this article is prohibited. 

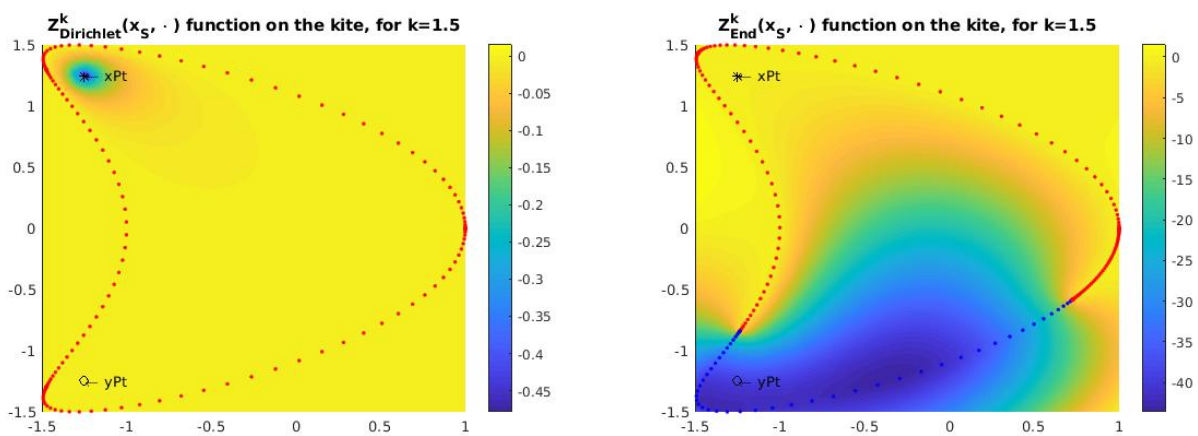

FIG. 5.3. The Zaremba function for $k_{\star}=2$ on the kite shape with Dirichlet boundary condition on the left and final mixed boundary conditions on the right. Further notation is as in Figure 5.1.
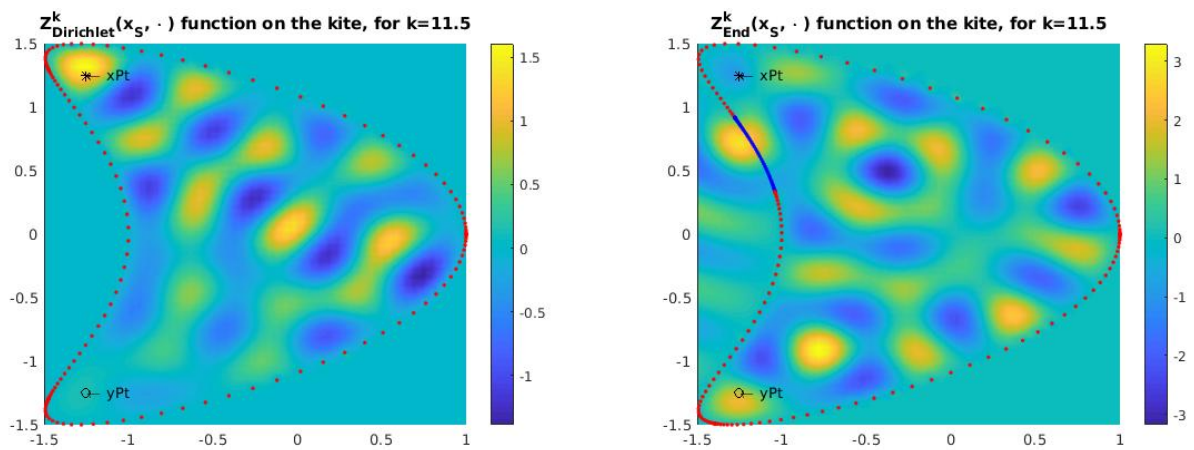

FIG. 5.4. The Zaremba function for $k_{\star}=11.5$ on the kite shape with Dirichlet boundary condition on the left and final mixed boundary conditions on the right. Further notation is as in Figure 5.1.

for $\tau \in[0,2 \pi)$. The target characteristic value is $k_{\star}=1.5$. The signal point $x_{\mathrm{S}}=$ $(-1.25,1.25)^{\mathrm{T}}$ and receiving point $y=(-1.25,-1.25)^{\mathrm{T}} . C_{\mathrm{tol}}=10^{-2}$ and $\varepsilon=0.05$. We remark here that the next higher Dirichlet characteristic value has multiplicity one and is at approximately 2.2099. The number of discretization points for the operator $\mathcal{A}(k)$ is $4 \cdot 48$. The result is displayed in Figure 5.3. The center of the Neumann boundary condition $\Gamma_{\mathrm{N}}$ is at $(-1.191,-1.493)^{\mathrm{T}}$ with length $\approx 3.119 . \mathrm{Z}_{\text {Dirichlet }}^{k}\left(x_{\mathrm{S}}, y\right) \approx$ $-4.05 \cdot 10^{-5}$ and $\mathrm{Z}_{\text {End }}^{k}\left(x_{\mathrm{S}}, y\right) \approx-39.38$.

In Figure 5.4, we have the same setup but for $k_{\star}=11.5$, with the next higher Dirichlet characteristic value around 11.6507. Here, the center of the Neumann boundary condition $\Gamma_{\mathrm{N}}$ is at $(-1.142,0.641)^{\mathrm{T}}$ with length $\approx 0.632 . \mathrm{Z}_{\text {Dirichlet }}^{k}\left(x_{\mathrm{S}}, y\right) \approx 0.148$ and $\mathrm{Z}_{\text {End }}^{k}\left(x_{\mathrm{S}}, y\right) \approx 1.68$.

\section{REFERENCES}

[1] E. Akhmetgaliyev and O. P. Bruno, Regularized integral formulation of mixed DirichletNeumann problems, J. Integral Equations Appl., 29 (2017), pp. 493-529, https://doi.org/ 10.1216/JIE-2017-29-4-493.

[2] E. Akhmetgaliyev, O. P. Bruno, and N. Nigam, A boundary integral algorithm for the Laplace Dirichlet-Neumann mixed eigenvalue problem, J. Comput. Phys., 298 (2015), pp. 1-28, https://doi.org/10.1016/j.jcp.2015.05.016. 
[3] H. Ammari, B. Fitzpatrick, H. Kang, M. Ruiz, S. Yu, and H. Zhang, Mathematical and Computational Methods in Photonics and phononics, Math. Surveys Monogr. 235, American Mathematical Society, Providence, RI, 2018, https://doi.org/10.1090/surv/235.

[4] H. Ammari, K. Imeri, AND W. Wu, A mathematical framework for tunable metasurfaces. Part I, Asymptotic Anal., 114 (2019), pp. 129-179.

[5] H. Ammari, K. Imeri, And W. Wu, A mathematical framework for tunable metasurfaces. Part II, Asymptotic Anal., 114 (2019), pp. 181-209.

[6] H. Ammari, K. Kalimeris, H. Kang, and H. Lee, Layer potential techniques for the narrow escape problem, J. Math. Pures Appl., 97 (2012), pp. 66-84.

[7] H. Ammari, H. Kang, and H. Lee, Layer potential techniques in spectral analysis, Math. Surveys Monogr. 153, American Mathematical Society, Providence, RI, 2009, https://doi. org/10.1090/surv/153.

[8] M. Sh. Birman and M. Z. Solomjak, Spectral Theory of Self-Adjoint Operators in Hilbert Space, D. Reidel Publishing Co., Dordrecht, 1987. Translated from the 1980 Russian original by S. Khrushchëv and V. Peller.

[9] A. Dabrowski, Explicit terms in the small volume expansion of the shift of Neumann Laplacian eigenvalues due to a grounded inclusion in two dimensions, J. Math. Anal. Appl., 456 (2017), pp. 731-744, https://doi.org/10.1016/j.jmaa.2017.07.027.

[10] N. Filonov, On an inequality for the eigenvalues of the Dirichlet and Neumann problems for the Laplace operator, Algebra i Analiz, 16 (2004), pp. 172-176, https://doi.org/10.1090/ S1061-0022-05-00857-5.

[11] D. S. Grebenkov And B.-T. NGuYen, Geometrical structure of Laplacian eigenfunctions, SIAM Rev., 55 (2013), pp. 601-667, https://doi.org/10.1137/120880173.

[12] E. M. Harrell, Geometric lower bounds for the spectrum of elliptic PDEs with Dirichlet conditions in part, J. Comput. Appl. Math., 194 (2006), pp. 26-35, https://doi.org/10. 1016/j.cam.2005.06.012.

[13] N. Kaina, M. Dupré, G. Lerosey, And M. Fink, Shaping complex microwave fields in reverberating media with binary tunable metasurfaces, Sci. Rep., 4 (2014), 6693.

[14] T. Kato, Perturbation Theory for Linear Operators, Springer-Verlag, Berlin, 1995. Reprint of the 1980 edition.

[15] A. Laptev, A. Peicheva, And A. Shlapunov, Finding eigenvalues and eigenfunctions of the zaremba problem for the circle, Complex Anal. Oper. Theory, 11 (2017), pp. 895-926.

[16] V. LotoreichiK AND J. RohLEDER, Eigenvalue inequalities for the Laplacian with mixed boundary conditions, J. Differential Equations, 263 (2017), pp. 491-508, https://doi.org/10.1016/ j.jde.2017.02.043.

[17] W. MCLean, Strongly Elliptic Systems and Boundary Integral Equations, Cambridge University Press, Cambridge, UK, 2000.

[18] S. Ozawa, Asymptotic property of an eigenfunction of the laplacian under singular variation of domains-the neumann condition, Osaka J. Math., 22 (1985), pp. 639-655.

[19] J. Saranen And G. Vainikko, Periodic Integral and Pseudodifferential Equations with Numerical Approximation, Springer Monogr. Math., Springer-Verlag, Berlin, 2002, https: //doi.org/10.1007/978-3-662-04796-5.

[20] K. Schmüdgen, Unbounded Self-Adjoint Operators on Hilbert Space, Grad. Texts in Math. 265, Springer, Dordrecht, 2012, https://doi.org/10.1007/978-94-007-4753-1.

Copyright $@$ by SIAM. Unauthorized reproduction of this article is prohibited. 